\title{
La construcción con tierra: un saber puesto al servicio de la comunidad de Cácota, Norte de Santander
}

\author{
Construction with land: knowledge put at the service of the \\ community of Cácota, Norte de Santander
}

Huber Giraldo G.*

Citar este artículo como: Giraldo, Huber. (2017). La construcción con tierra: un saber puesto al servicio de la comunidad de Cácota, Norte de Santander. Revista Nodo, 12(23), 20-30

\section{Resumen}

La formación de trabajadores de la construcción, profesionales y comunidad en general en tecnologías alternativas y tradicionales de construcción como la que utiliza tierra en sus diferentes técnicas, mediante metodologías que comparten el conocimiento de manera horizontal como el "aprender haciendo", responde al problema urgente de formar técnicos capaces que contribuyan en la conservación del patrimonio edificado y en recuperar y dar continuidad a sistemas constructivos tradicionales y probados, que permitan atender el déficit de viviendas y la configuración de un hábitat sostenible.

Palabras clave: Taller de Construcción con tierra. Tecnología alternativa y apropiada de construcción. Diseño participativo.

\section{Abstract}

The training of construction workers, professionals and the community in general in alternative and traditional construction technologies such as the one that uses land, in its different techniques, through methodologies that share knowledge horizontally as "learning by doing", responds to the problem urgent to train capable technicians in the conservation of the built heritage, to the need to recover and give continuity to traditional and tested construction systems, which allow to address the housing deficit and the construction of a sustainable habitat.

Keywords: Construction workshop with earth. Alternative and appropriate construction technology. Participatory design.

Fecha de recepción: 23-04-2017 • Fecha de aceptación: 4-10-2017

\footnotetext{
* Arquitecto. Docente e Investigador de la Facultad de Ingenierías y Arquitectura. Programa de Arquitectura. Universidad de Pamplona, Pamplona, Norte de Santander, Colombia. Magíster en Gestión Urbana, Universidad Piloto de Colombia. PhD en Desarrollo Urbano Sustentable de la Universidad Politécnica de Madrid. Madrid. España. Director del grupo de investigación URBANIA. Miembro fundador de la Corporación Ciudad Taller. Correo electrónico: observatoriourbano@gmail.com , hgiraldogiraldo@gmail.com , corporaciónciudadtaller@gmail.com
} 


\section{Sustentabilidad y construcción con tierra}

Si se entiende la arquitectura sostenible como la iniciativa que busca hacer posible que el futuro emergente sea ecológicamente sano y habitable, optimizando los recursos naturales y el aprovechamiento de las condiciones climáticas, de materiales y geográficas del entorno, haciendo un uso eficiente de estos recursos y disminuyendo al máximo el impacto ambiental, la arquitectura de tierra posee las características de la construcción sostenible en lo que respecta al ahorro de energía y la disminución de la huella ecológica, ya que su puesta en obra -en estado natural- no requiere ningún proceso de transformación.
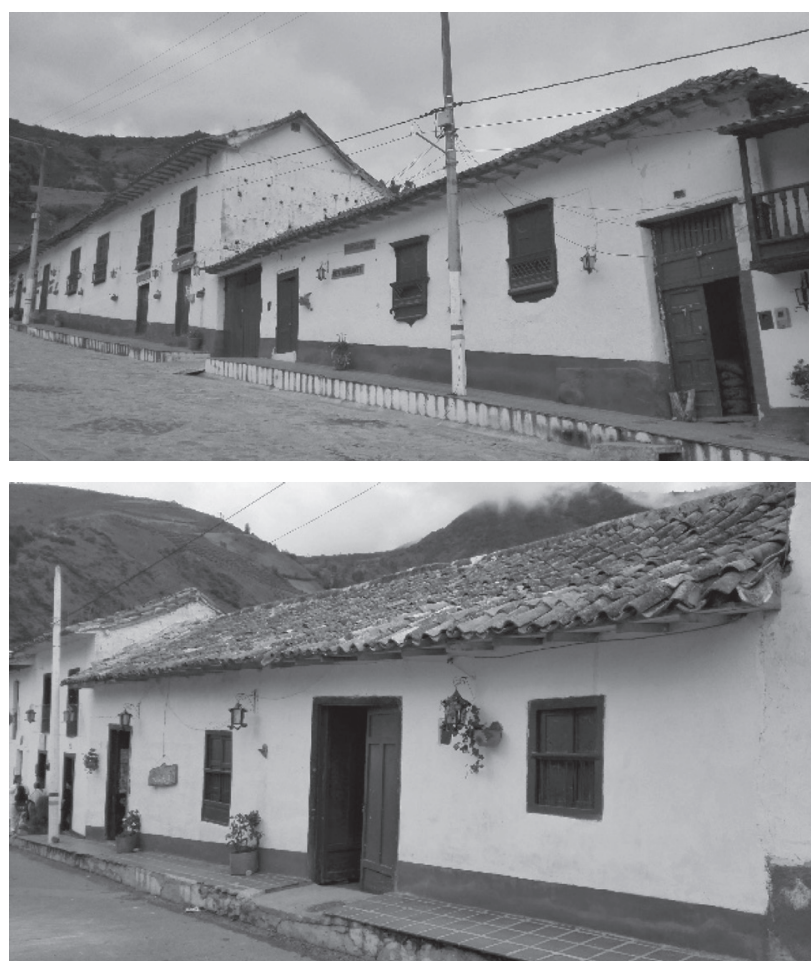

Figuras 1 y 2 . Arquitectura patrimonial de Cácota. Fuente: propia

Efectivamente, no precisa cocción por lo que ahorra una importante cantidad de energía y además, no produce contaminación ambiental. El material es económico, de fácil puesta en obra y no requiere grandes costos de transporte ni manipulación, ya que generalmente se encuentra disponible de calidad aceptable en el mismo lugar donde se pretende construir. Tampoco emite $\mathrm{CO}_{2}$ a la atmósfera, ni en su proceso de producción, ni en su transporte; por otra parte, el barro es reutilizable pues basta con triturarlo y humedecerlo con agua para volver a usarse.

Las labores de construcción con tierra han sido transmitidas tradicionalmente de generación en generación desde las antiguas civilizaciones hasta hoy. No obstante, por diversas razones entre las cuales se incluye el avance de la industrialización y la incorporación de nuevos materiales como el concreto reforzado, junto con el desconocimiento y falta de valoración de las técnicas tradicionales de construcción con tierra, incentivaron el uso de otros materiales y ayudaron de paso a instalar una serie de prejuicios en torno a la técnica de construcción con tierra a nivel social, cultural y estético.
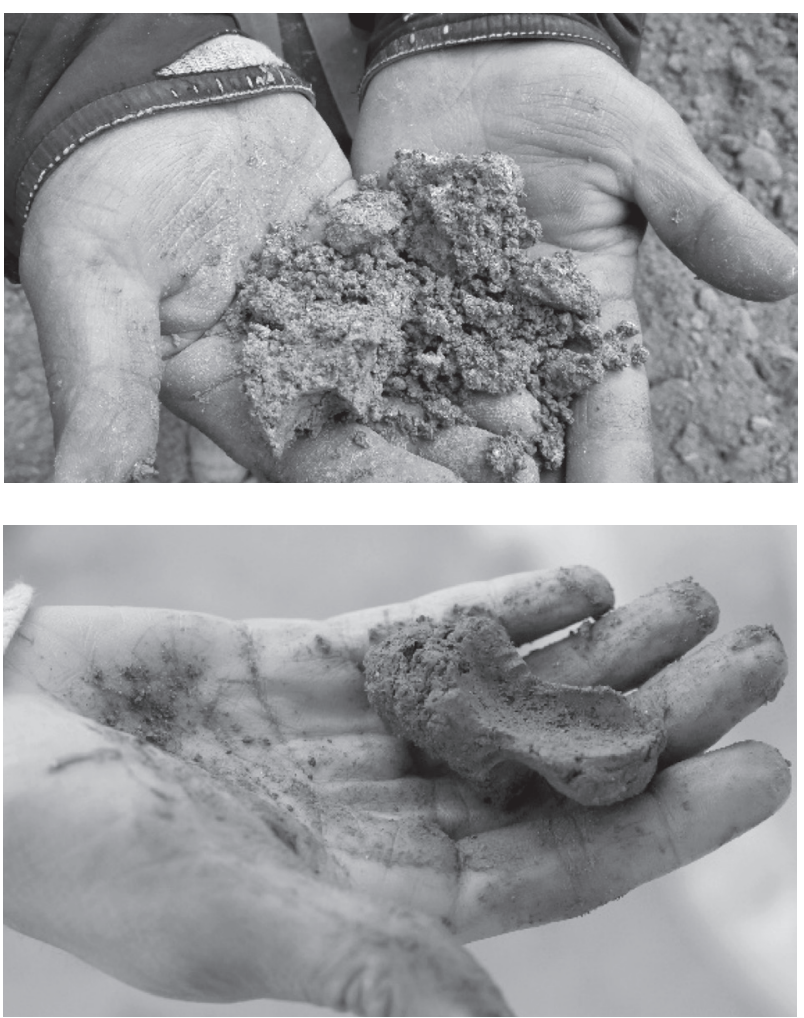

Figuras 3 y 4. Talleres de construcción con tierra cruda. Caracterización de la tierra. Fuente: propia 
Los centros históricos de muchos poblados y sus construcciones rurales poseen manifestaciones de la arquitectura local de carácter patrimonial y vernácula que confieren identidad cultural y constructiva a sus habitantes; sin embargo dichos entornos enfrentan problemas como el abandono, la destrucción y la pérdida de actividades humanas tradicionales, la construcción acelerada de nuevos inmuebles edificados con técnicas convencionales basadas en materiales industrializados y de alto costo.

La Corporación Ciudad Taller desarrolla actividades en el dominio de la arquitectura y las técnicas de construcción y diseño con tierra. Éstas comprenden cursos referidos a la enseñanza y formación de colectivos ciudadanos en el conocimiento y aplicación de técnicas sostenibles de construcción y en la materialización de propuestas de hábitat y conocimiento de sistemas constructivos.

Ciudad Taller se plantea un objetivo fundamental: reforzar la actividad de rehabilitación, mantenimiento de la arquitectura tradicional vernácula y colonial, junto a la generación de nuevas arquitecturas con fundamento en técnicas tradicionales, alternativas y apropiadas de construcción como factor de desarrollo sostenible (social, económico y medio ambiental). Dicha estrategia permite a los gobernantes, profesionales y técnicos de construcción plantear iniciativas de promoción de nuevas arquitecturas contextuales, de restauración y rehabilitación de arquitecturas locales al disponer de un marco de referencia, ayudándoles a perfeccionar y mejorar sus capacidades y conocimientos.

La Corporación Ciudad taller colabora con las instituciones gubernamentales y la comunidad en general en la conservación del patrimonio edificado y en procesos de responsabilidad social, aportando formación mediante prácticas pedagógicas en colaboración con expertos para su protección y para el desarrollo de nuevos proyectos constructivos fundamentados en la tradición cultural, que agregan valor al amplio y diverso patrimonio compartido.
Ante la creciente urbanización del territorio nacional, los centros urbanos tradicionales como el del Municipio de Cácota, vienen siendo objeto de procesos crecientes de transformación que no tienen en cuenta las condiciones de tenencia y su riqueza cultural. Sin duda, este municipio con alta tradición en la posesión de arquitecturas vernáculas y patrimoniales tiene un reto enorme: respetar la memoria colectiva vinculada al entorno construido y heredado, asegurando la re-funcionalización de sus conjuntos edilicios en asocio con la incorporación de nuevas creaciones espaciales que articuladas a su tradición constructiva, le den continuidad a dicho valor.
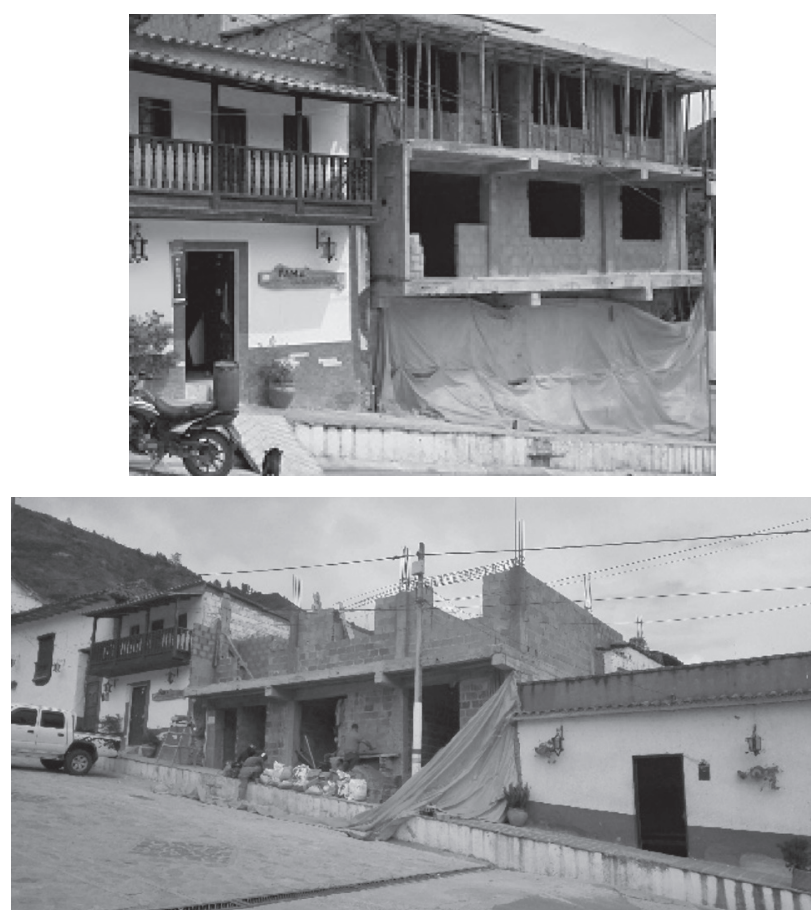

Figuras 5 y 6. Nueva construcción con cemento y ladrillo que cambia la identidad cultural y constructiva del pueblo. Cácota. Fuente: propia

El Municipio de Cácota cuenta con un valioso patrimonio edificado que incorpora técnicas y sistemas que utilizan la tierra natural como material de construcción, contando con importantes edificios tanto de su patrimonio histórico y monumental como residencial, y prácticamente la totalidad de los edificios del patrimonio tradicional rural. En 
tal sentido, la conservación de este patrimonio requiere mantener vivas sus técnicas constructivas junto con la investigación aplicada impartida por expertos que aportan mayor conocimiento sobre las bondades del material y su redescubrimiento desde ópticas de arquitectura bioclimática y sustentable.

Recientemente el Municipio de Cácota reconoce en su patrimonio inmueble uno de sus principales valores y elementos de atracción de visitantes y del turismo cultural y ha comenzado a clasificarlo y a establecer normas que permitan proteger sus amplias zonas homogéneas poseedoras de arquitecturas vernáculas y bienes de interés cultural.

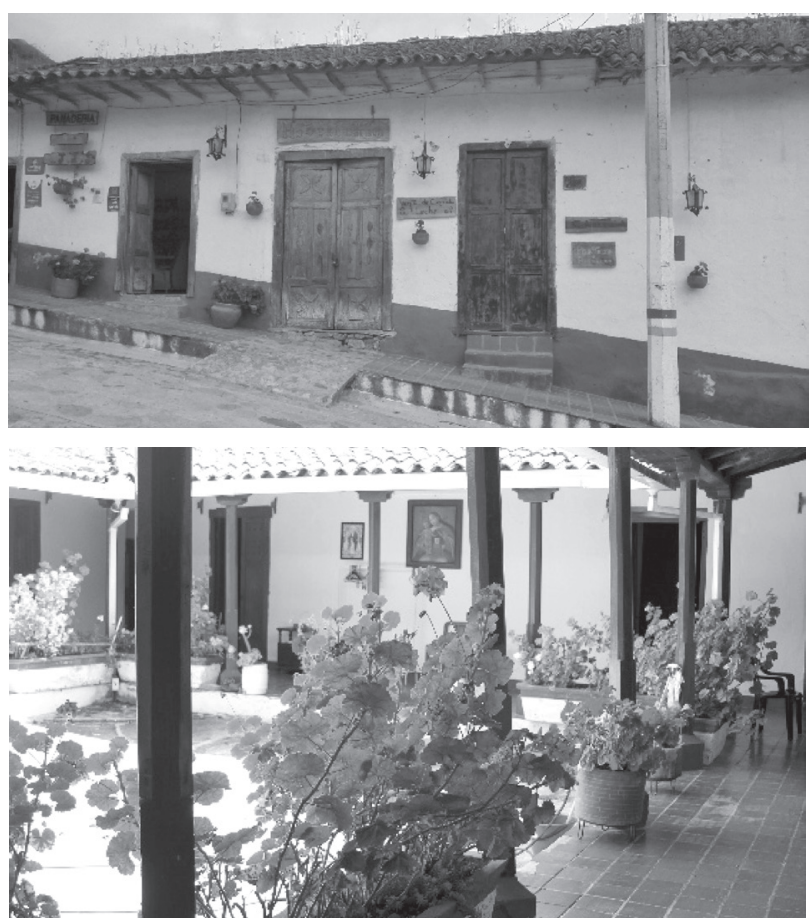

Figuras 7 y 8. Patrimonio arquitectónico de Cácota en calles e interiores. Fuente: propia

En tal sentido, la conservación del centro histórico por parte de entes gubernamentales locales y la puesta en práctica de sus planes de protección depende en buena medida de la vinculación de la comunidad mediante procesos de participación ciudadana, reforzada con la difusión de conocimiento y la formación de técnicos y constructores capacitados en la provisión y praxis de técnicas tradicionales y mejoradas de construcción con tierra. Desde esta perspectiva es viable el inicio del proceso de mejoramiento de modelos locales de construcción orientados a la protección de la tradición constructiva, los bienes de interés cultural y la generación de nuevo patrimonio entre residentes y propietarios permanentes.

Gracias al valioso apoyo de profesionales y comunidad local, ha resurgido el interés por el uso de este material y el resguardo del patrimonio arquitectónico local, que en gran parte se encuentra en peligro de desaparición y que está construido en diferentes técnicas de tierra (bahareque, adobe, bloque y tapia pisada, entre otras). Es dentro de este contexto que se requiere reactivar la cadena de revalorización y difusión de la tierra como material de construcción.

En cumplimiento de los valores misionales de la Corporación Ciudad Taller se desarrolló un convenio con la Alcaldía de Cácota para realizar un taller en Construcción con tierra, cuyo objetivo fue transferir conocimientos de técnicas tradicionales, alternativas y apropiadas de construcción con tierra mediante estrategias y métodos de aprendizaje de fácil acceso con modelos de enseñanza-aprendizaje como "aprender haciendo", en comunidades que habitan entornos vernáculos y patrimoniales construidos con este material que deben ser conservados, promovidos y creados, mediante procesos de producción social del hábitat.

Los cursos impartidos en la modalidad teóricopráctico tienen los siguientes objetivos:

- Identificar, acopiar, tipificar y transmitir estrategias y métodos de aplicación de técnicas tradicionales, alternativas y apropiadas de construcción con tierra mediante procesos demostrativos de enseñanza para una eficaz conservación, restauración y construcción de viviendas con tierra como material central.

- Formar recursos humanos, mediante un sistema de enseñanza-aprendizaje de carácter teórico, práctico y demostrativo de los oficios tradicionales, alternativos y mejorados, bajo criterios de responsabilidad normativa y sismo-resistencia, con base en el conocimiento, la investigación y 


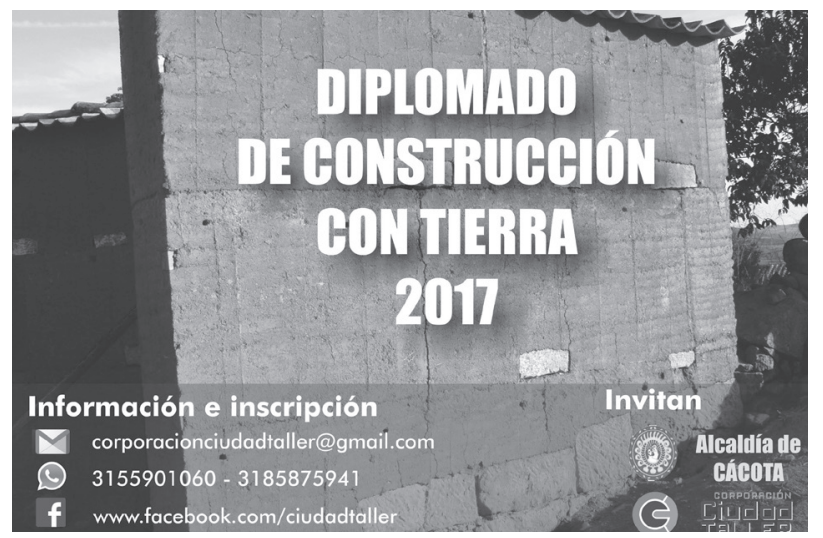

la experimentación, desarrollada y provista por expertos e investigadores de reconocimiento nacional e internacional en diferentes sistemas constructivos de tierra, tales como: tapia pisada tradicional, tapia pisada alternativa, de espesores mínimos, bahareque, bloque de tierra comprimida y acabados en arcillas.

- Rescatar y difundir conocimientos en técnicas constructivas tradicionales y mejoradas de construcción con tierra entre comunidades, técnicos y profesionales como una de las formas más adecuadas de promover el crecimiento social y cultural de la arquitectura patrimonial, en la perspectiva de adoptar formas de construcción sostenibles, que puedan realizar procesos idóneos de conservación, de nuevas edificaciones y viviendas.

- Promocionar formas constructivas ecológicamente sostenibles y más económicas, ante el predominio de la construcción basada en materiales que demandan en su producción el consumo creciente de combustibles fósiles y no renovables, con la generación de una alta huella ecológica en su producción, además de su alto costo económico.

- Involucrar al ser humano y al medio ambiente que lo rodea como parte de su solución habitacional, desde técnicas de construcción con tierra estabilizada, como la tapia pisada, el bloque de tierra comprimida, el bahareque y acabados con arcillas naturales, son auspiciadas como estrategia para el desarrollo de nuevas propuestas constructivas.
- Iniciar y formar colectivos ciudadanos, maestros de obra, obreros, estudiantes de ingenierías y arquitectura, profesionales de diferentes disciplinas en tareas de investigación y extensión, en el área específica de la producción de técnicas de construcción con tierra. La formación ofrecida está basada en la comprensión y el mejoramiento continuo de formas físicas de una cultura de mayorías, expresado en el ambiente edificado, en sus necesidades y valores, así como en los deseos.

\section{Los talleres}

Estos ejercicios introducen a los participantes en las propiedades de la tierra y luego desarrolla sus técnicas constructivas y las terminaciones o acabados de tierra. Asimismo, contribuyen a fomentar la necesidad de ver las técnicas de tierra nuevamente valoradas por sus amplias cualidades de sostenibilidad; donde los fundamentos y principios de las técnicas de construcción con tierra, se ajustan a los conceptos de ecología y sostenibilidad en la arquitectura, contando con su interrelación con el patrimonio natural, salud, clima, recursos
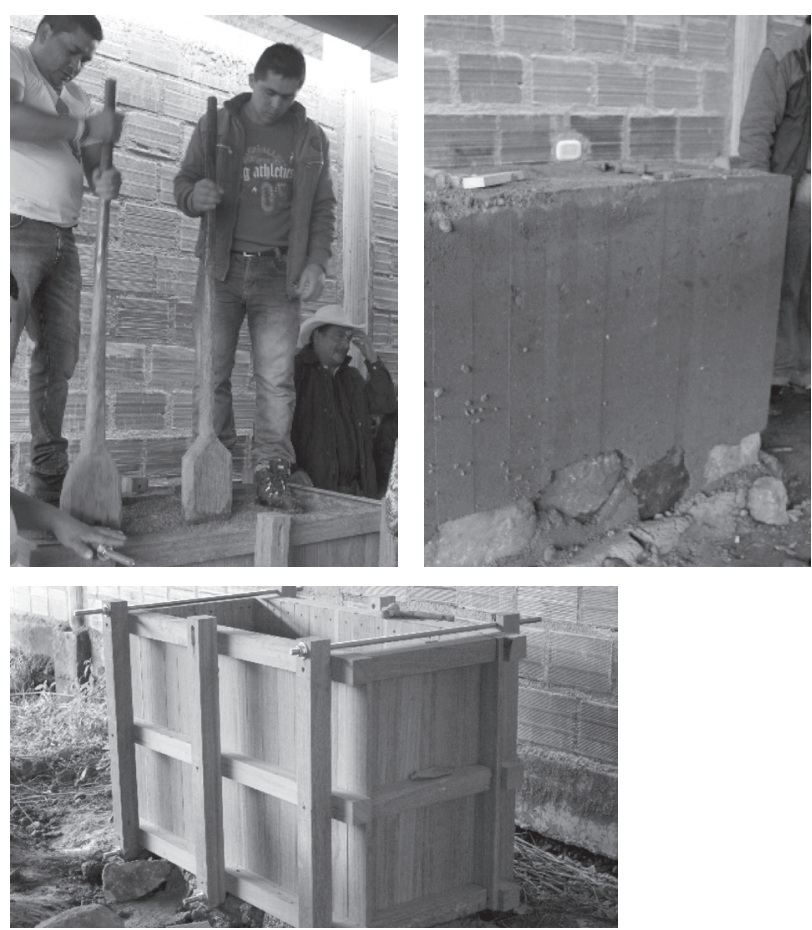

Figuras 9, 10 y 11. Taller de tapia pisada. Fuente: propia 
naturales, sociales y culturales, proporcionando las bases necesarias para su aplicación, diseño y construcción.

El taller de construcción con tierra parte del principio pedagógico "aprender haciendo" y además del respaldo teórico en cada uno de los temas, cuenta con un importante componente práctico de ejercicios demostrativos donde se observan las particularidades y detalles de los sistemas constructivos y las especificaciones técnicas que se complementan con ejercicios de diseño participativo en terrenos propios del Municipio.

El alcance de los conocimientos sobre las técnicas constructivas, está mediado por varias situaciones: de una parte, el peso significativo del componente artesanal en las técnicas constructivas; por la otra,

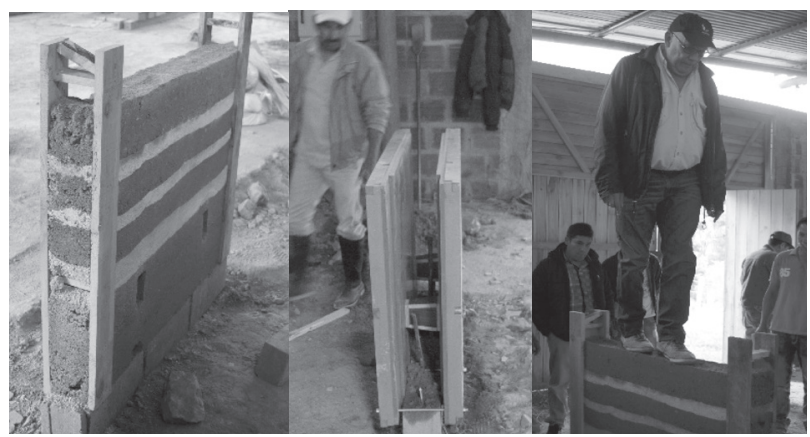

Figuras 12, 13 y 14. Taller de tapia alternativa $(15 \mathrm{cms}$ de espesor). Fuente: propia
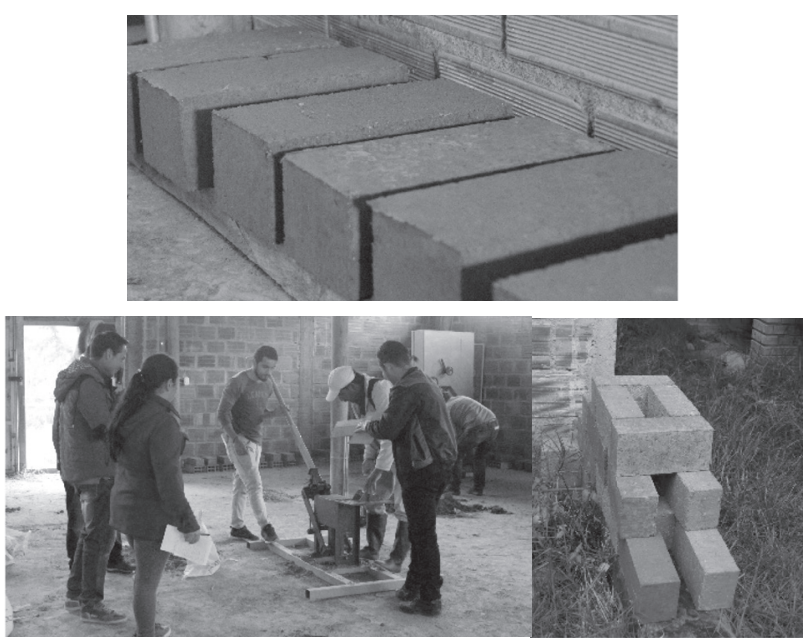

Figuras 15, 16 y 17. Taller de bloque de tierra comprimida (BTC) con el uso de la prensa CinvaRa. Fuente: propia su técnica mejorada desde la investigación suministrada por los mejores expertos; y por último, por el conjunto de normas y su regulación que deben ser tenidas en cuenta en los procesos constructivos.

La comunidad al conocer las técnicas constructivas con tierra, sus potencialidades formales, ambientales y de costos, está en condiciones de proyectar un espacio habitable con cometidos de alta intencionalidad y de expresión cultural. En tal sentido esta experiencia puede catalogarse como un conocimiento indispensable, ya que va desde lo elemental hasta llegar a ser un conocimiento altamente especializado.

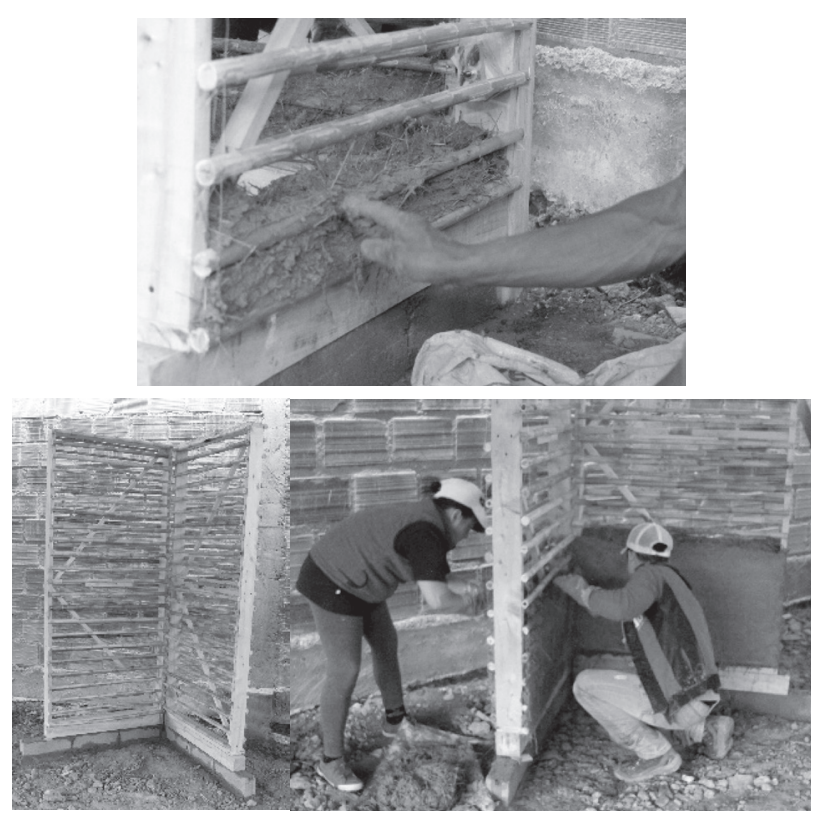

Figuras 18,19 y 20. Taller de bahareque. Fuente: propia

La arquitectura en tierra, desde esta perspectiva, no implica una situación ideal o de ensueño, sino una realidad que crece cada vez más dadas las ventajas que presenta, junto con las realidades en términos de costos constructivos, bajo impacto ambiental asociado a las demandas crecientes del abastecimiento energético actual y futuro. Elementos básicos que constituyen la construcción del espacio arquitectónico y cultural, configurando un conjunto significativo soportado en las costumbres de uso tradicional, cultural y de innovación. 

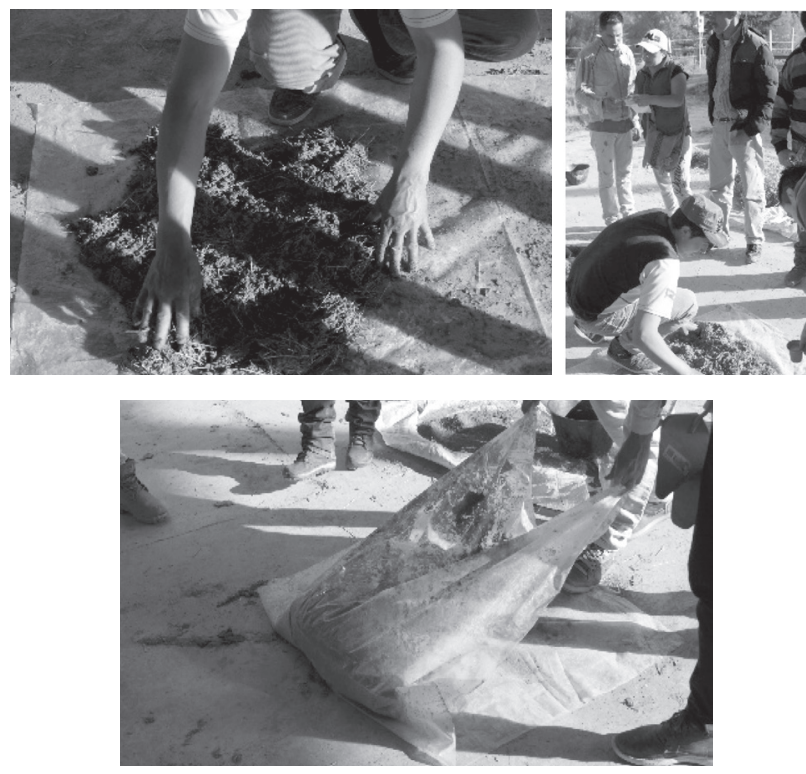

Figuras 21, 22 y 23. Taller de acabados arquitectónicos con arcilla. Fuente: propia

\section{Transferencia de técnicas en la arquitectura en tierra}

La transferencia de técnicas de construcción y diseño con tierra consiste en la transmisión de saberes, prácticos y de información entre especialistas, investigadores, profesionales, activistas, diseñadores y constructores, colectivos o comunidades que requieren o demandan dicha formación. Dicha transferencia de conocimientos se realiza sobre activos intelectuales que poseen los especialistas y que han adquirido mediante procesos de exploración, investigación y ensayo que desean transmitir a las comunidades.

La transferencia de saberes es una alternativa para difundir experiencias constructivas probadas y conocimientos producto de la investigación que garanticen su reproducción total o parcial y su posterior aplicación en procesos constructivos y de diseño propio o su implantación en procesos productivos ampliados, como proyectos de vivienda masivos y/o de autoconstrucción.

Las técnicas de construcción con tierra se convierten así en un elemento estratégico para las instituciones gubernamentales, ONGs y de autoconstrucción de las comunidades ya que su gestión es clave para la creación y consolidación de ventajas formativas y competitivas en un sector como el de la construcción, dotándolo de técnicas alternativas y apropiadas.

Se entiende, que los procesos de formación tecnológica se revierten en ideas esenciales para procesos creativos de cómo se diseña y se construye, así como para la incorporación paulatina de oportunidades de innovación tecnológica basadas en sistemas tradicionales mejorados, poseedores de un arraigo tradicional y asociados a prácticas vernáculas de construcción, que les permite construir su propia vivienda y las edificaciones que la comunidad necesita en cooperación o por autoconstrucción.

La tecnología emergente de construcción con tierra cruda es impartida a los participantes como fruto de la actividad de investigación y desarrollo tecnológico adquirido por los expertos, que permite las innovaciones técnicas que demandan las comunidades y logran dar respuesta a sus necesidades de vivienda y de construcción económica.
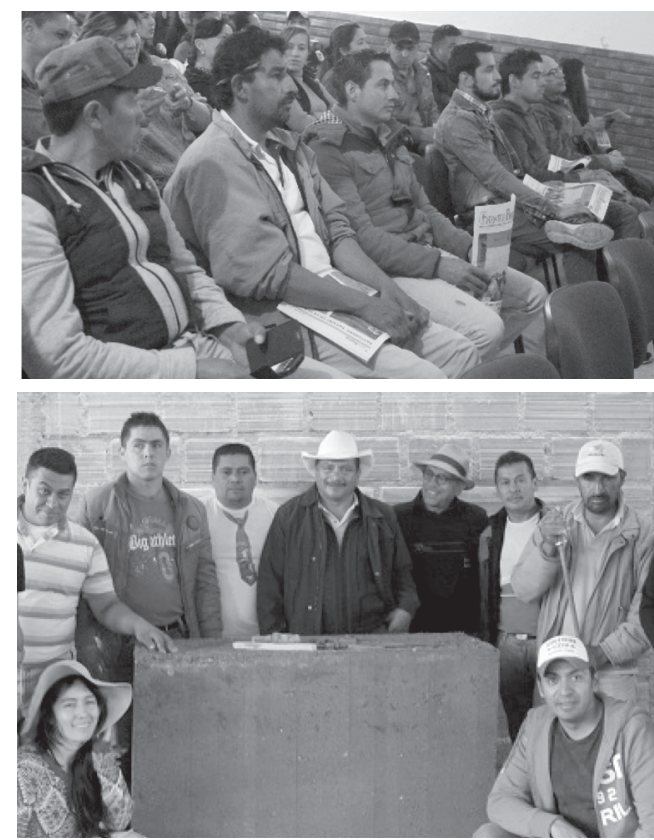

Figuras 24 y 25. Participantes del taller de construcción con tierra cruda. Fuente: propia 


\section{Los talleres de diseño participativo}

La Alcaldía de Cácota promueve un diálogo franco y abierto con la ciudadanía mediante la creación, como política social, de un programa de vivienda basado en el protagonismo de los ciudadanos, en su diseño y ejecución, apoyado en la capacitación recibida por integrantes de la comunidad en técnicas alternativas de construcción con tierra que les permite hacer su contribución técnica.

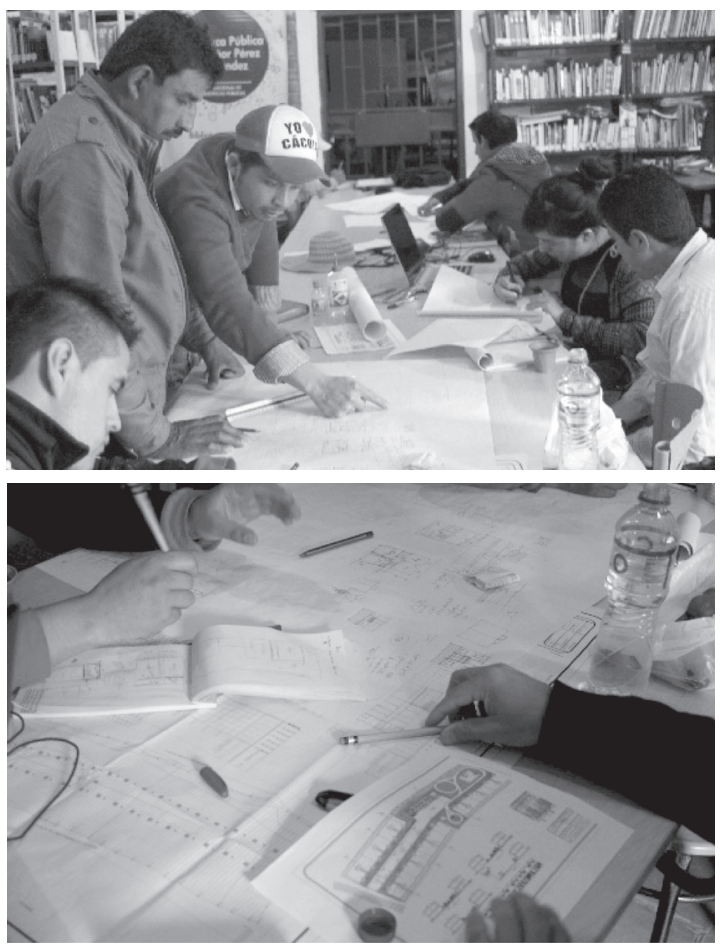

Figuras 26 y 27. Taller de diseño participativo. Diseño de Conjunto de Vivienda de Interés Prioritario en Tierra. Fuente: propia

Mediante los talleres se promueve un acercamiento a la comunidad, un intercambio de conocimientos de los procesos productivos y sistemas constructivos practicados por ésta, el reconocimiento de su medio ambiente y la verificación de los cambios que vienen afectando a su arquitectura, sus espacios y bienes, para luego adaptar las diferentes estrategias de formación así como las técnicas de construcción ajustadas a su cultura y al medio ambiente con el fin de aumentar su adaptabilidad y disminuir su vulnerabilidad.
De esta manera se impulsa la producción social del hábitat y el desarrollo comunitario, con el seguimiento a los procesos constructivos y su aplicación a diferentes componentes del proyecto, que mediante acciones colaborativas como la toma de decisiones en consenso y la ayuda mutua en los procesos constructivos y productivos, permitirán la autogestión y la producción colectiva de diseño y propuestas de intervención basados en el uso de la tierra. Todo esto contribuye al desarrollo comunitario.

Enfocados en el tema de la vivienda social, la comunidad, los trabajadores de la construcción y participantes de los talleres, con la dirección de profesionales e investigadores, resuelven adecuadamente no solo la implementación de técnicas alternativas y apropiadas de construcción en modelos de diseño y prototipos, con fundamento en el lenguaje de las tradiciones constructivas heredadas, sino que asumen con propiedad la solución de un hábitat integral y sostenible para sus conciudadanos.

Se trata de un proceso de producción social de vivienda en centros históricos urbanos y patrimoniales, como un proceso de ideación que se realiza con la participación activa de la comunidad en alianza con otros agentes sociales (instituciones, ONGs, profesionales), que operan sin ánimo de lucro, promoviendo las capacidades de autogestión y de protagonismo ciudadano, que dan prioridad al valor de uso colectivo, a los intereses y necesidades de la comunidad, a los valores sociales y culturales, a la memoria urbana colectiva, por encima del valor comercial o de intereses particulares que puedan generar las acciones privadas de producción de vivienda

En tal sentido, el colectivo de participantes en los talleres de construcción con tierra y de manera continua asume mediante sesiones de diseño participativo e interdisciplinar (con el apoyo de investigaciones y aplicaciones prácticas de éxito probado), posibles soluciones de vivienda que podrán dotarse con éxito, en un claro ejemplo del verdadero desarrollo humano de su comunidad. 


\section{El proyecto de construcción de vivienda con tierra}

Con recursos económicos y la donación de un terreno urbanizado por parte de la Alcaldía de Cácota junto con la comunidad, se vienen construyendo hoy un conjunto de 18 viviendas en tierra, mediante la aplicación de los conocimientos impartidos por La Corporación Ciudad Taller y sus especialistas invitados, en el Diplomado de construcción con tierra y el diseño participativo; viviendas que serán donadas de manera gratuita al mismo número de familias que hoy viven en condiciones de hacinamiento.

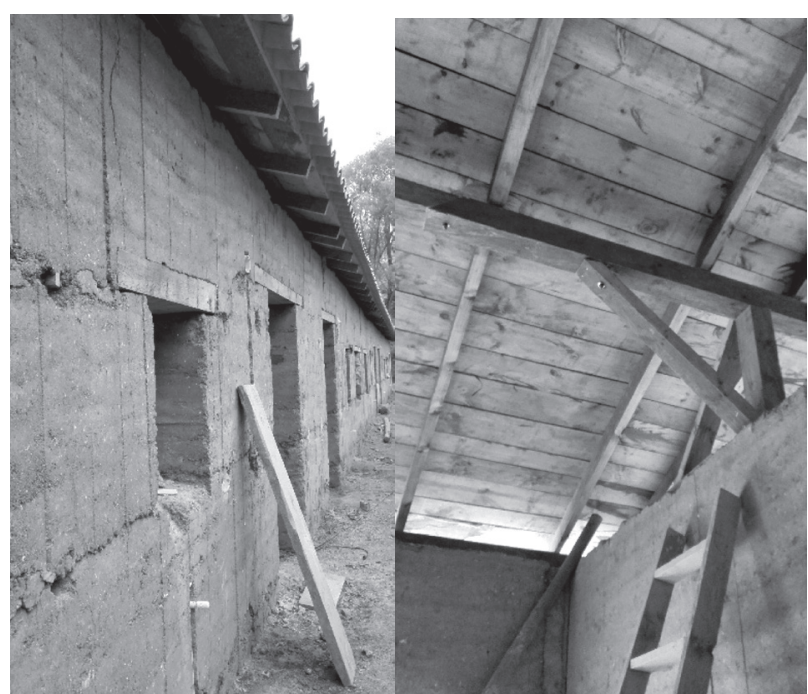

Figuras 28 y 29. La construcción de viviendas en tapia pisada, bloque de tierra comprimida y techumbre en madera de pino pátula y teja de barro. Fuente: propia

Beneficiarios, técnicos formados en técnicas de construcción con tierra, voluntarios y la comunidad vienen colaborando en dicho proyecto con una inversión económica muy baja, aprovechando de manera esencial los recursos materiales existentes en el entorno y con la disposición de vincular la recuperación de valores y expresiones propias de la cultura del lugar. Todo es construido bajo los principios de "lo que hay", con materiales del lugar, haciendo uso de la sabiduría local con los aportes de las técnicas tradicionales mejoradas que valoran la expresión arquitectónica local, lo ancestral, en un contexto contemporáneo. El convenio interinstitucional entre la Alcaldía de Cácota y Ciudad Taller mediante un curso impartido a la comunidad, tuvo en esta oportunidad la afortunada posibilidad de trabajar en un encargo real y colectivo. Así, los participantes debieron trabajar no solo con el diseño de las viviendas,

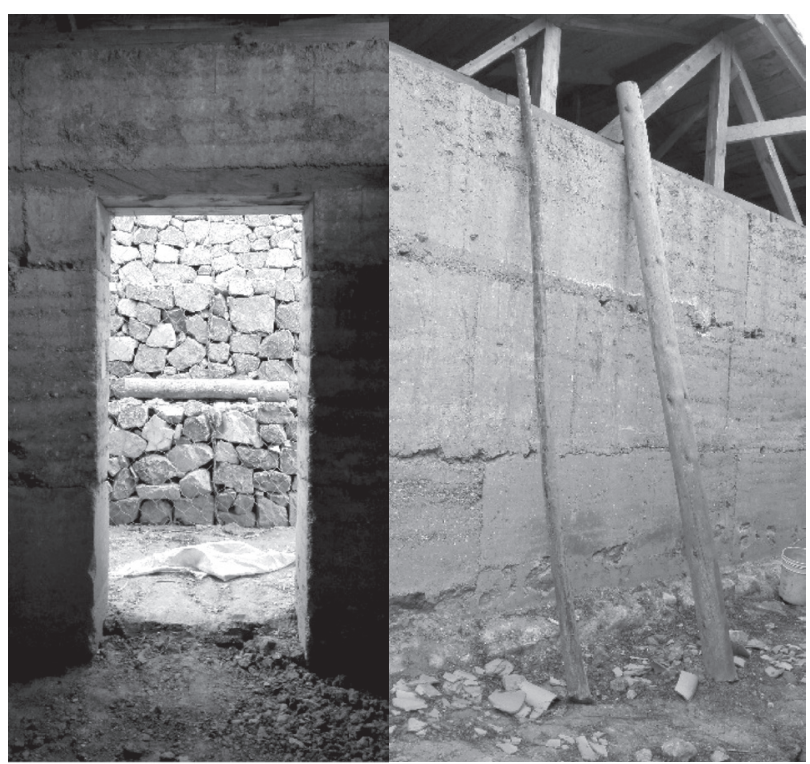

Figuras 30 y 31. Cerramiento exterior y muros divisorios en tierra, de las viviendas. Fuente: propia

sino también con presupuestos y la construcción de las viviendas.

Aquí no se ve la arquitectura como un producto sino como la oportunidad de construir comunidad, aunar esfuerzos en la construcción de una sociedad de participación y solidaridad, que trabaja por la herencia cultural y su puesta a punto en la actualidad, mediante una arquitectura que es construida por y para todos. Un excelente ejemplo donde se aborda la identidad formal colectiva con un proyecto de vivienda construido con tierra donde la historia cultural se transforma en vivienda gratuita y en aprendizaje. Un proyecto social que beneficia a comunidades desfavorecidas. Una construcción colectiva que refuerza lo comunal, materializa la memoria colectiva y la vivienda gratuita. 


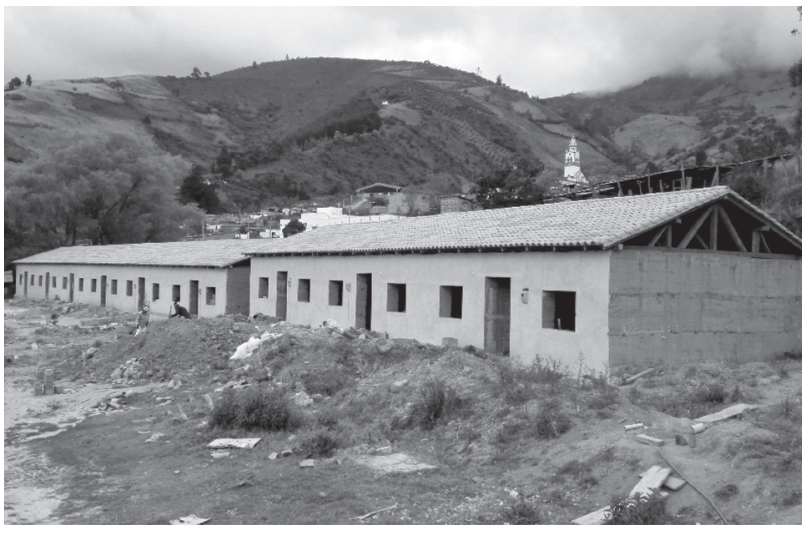

Figura 32. Conjunto de viviendas en construcción en Cácota. Construidas en tierra, con el apoyo de la Alcaldía y la capacitación en técnicas de construcción con tierra, impartido a miembros de la comunidad. Fuente: propia

\section{Conclusiones}

Municipios tradicionales con significativa presencia de arquitectura vernácula y patrimonial heredada, generalmente alejados de los grandes centros urbanos, vienen siendo objeto de la introducción de nuevas actividades productivas motivadas especialmente por el turismo y la oferta de servicios, generando una acelerada expansión urbana y constructiva que se traduce en el deterioro de las arquitecturas heredadas y en la transformación del espacio físico-ambiental de los poblados. Todo ello acompañado de una falta de normativa referida a la protección del patrimonio construido y a la planificación urbana, que propicie edificaciones orientadas tanto a la preservación como a la generación de nuevos patrimonios regionales en una producción de tejidos urbanos acordes con el crecimiento poblacional y la identidad local. Por tanto es necesario proveer estrategias que permitan identificar los tipos de edificación que son expresión de la permanencia y vigencia de las tradiciones constructivas con tierra e igualmente las zonas urbanas homogéneas que en conjunto las agrupan, así como su distribución y localización para protegerlas, conservarlas o mejorarlas ante el riesgo de ser alteradas y modificadas.

El conocimiento de la arquitectura local, los materiales y su lógica constructiva constituye el fundamento necesario para hacer nueva y mejor espacialidad. Con lo cual, se podrán promover desarrollos urbanos y arquitectónicos con aplicación de tecnologías apropiadas y mejoradas que den continuidad, permanencia y vigencia a las tradiciones constructivas que emplean la tierra, en armonía con el contexto urbano y el área geográfica de los poblados.

Una arquitectura adaptada al contexto es resultado de la adecuada interpretación de la cultura local y de la sociedad que le acoge, por lo tanto emerge de la acción y expresión colaborativa y colectiva, no de un acto individual. En este sentido, la expresión arquitectónica local es expresión del bien común, se adecúa al contexto local, es evolutiva, agrega valor a la cultura heredada expresando una arquitectura cuya estética se fundamenta en la interpretación de su cosmogonía, ingrediente central para un hábitat de calidad, muy escaso en la arquitectura actual y globalizada.

La creciente demolición del patrimonio arquitectónico construido, la pérdida de conocimiento de las prácticas constructivas tradicionales de tierra y la tímida incorporación de la investigación en el fortalecimiento, mejoramiento y optimización de los sistemas constructivos tradicionales además de la imposibilidad de proveer nuevas arquitecturas y propuestas constructivas con aplicación de la tierra como material central, hacen evidente la importancia de instaurar programas de investigación, de formación práctica y teórica en uso del suelo en el diseño, la restauración y la construcción arquitectónica, que provoquen impacto positivo en la expresión arquitectónica, la eficiencia energética y el desarrollo sostenible en los poblados regionales.

Mediante el abordaje integral del diseño de viviendas por parte de los participantes del taller, se tienen en cuenta tanto las soluciones técnicoconstructivas aprendidas como las variables de un desarrollo sostenible, ya que se asume el proceso de mejoramiento del hábitat y la vida de las personas como un recorrido en el que son importantes las técnicas alternativas de uso energético y los mecanismos efectivos de aprovechamiento de 
recursos naturales existentes. En este sentido, el desarrollo de una arquitectura local retoma saberes tradicionales y nuevos conocimientos desde la conciencia social en una relación de reciprocidad e intercambio con la naturaleza, acomodándose al conocimiento profundo del contexto.

$\mathrm{Al}$ encarar la concepción de la vivienda no como un problema de cantidad sino como un proceso cultural, económico, social y tecnológico, se supera la sumisión a tecnologías foráneas y dominantes, las de materiales industrializados, para dar paso a prácticas constructivas que asumen las dificultades de ampliación y mejora de la vivienda como un compromiso consecuente de los habitantes con su propia casa. Se fortalecen procesos de organización social y de creación colectiva que enriquecen el hábitat de las comunidades, se intercambian experiencias, capacidades y conocimientos entre los participantes desde la condición de habitante hasta la de constructor y de profesional.

Es necesario trabajar por desmentir los mitos generalizados que asocian pobreza y deterioro de las viviendas y su inestabilidad sísmica al material constructivo y valorar las formas culturales de habitar y vivir de las comunidades, mediante el uso y apropiación de este noble material. También es imprescindible promover y consolidar normativas que valoren el entorno y permitan la construcción con tierra y, al mismo tiempo, difundir y fortalecer las acciones de formación hacia la concepción de sistemas constructivos con este material basados en la investigación y en tecnologías mejoradas que ofrezcan seguridad a la población. Y, por último, educar y explorar los horizontes de la técnica y su diseño para ofrecer amplias alternativas creativas y posibilidades de desarrollo sostenible. 